\title{
UN ANÁLISIS DEL CRECIMIENTO Y LA CONVERGENCIA DE LA ECONOMÍA ANDALUZA ENTRE 1975 Y 1995
}

José GARCÍA ROA*

\section{INTRODUCCIÓN}

En este trabajo partimos de la base de que la evidencia empírica no se decanta por ninguna de las dos teorías genéricas del crecimiento ${ }^{1}$, sino que, más bien, el resultado empírico más cercano a uno u otro enfoque depende de multitud de variables como son: la movilidad de los factores, la tendencia creciente, decreciente o constante de los rendimientos, el nivel de conocimientos, la apertura al exterior, etc. En la actualidad están siendo objeto de intensa investigación los mecanismos a través de los cuales estos factores influyen en el crecimiento.

A tal efecto, a continuación se estudian las circunstancias evolutivas de la región en las dos décadas recientes, haciendo especial referencia a la evaluación de las características estructurales que tienen una mayor incidencia en las tasas de crecimiento y en los niveles de desarrollo.

El relativo retraso con que se publican los datos referidos a las principales magnitudes a escala provincial, nos ha obligado a considerar, fundamentalmente, los cambios producidos entre 1975 y 1995. Esta circunstancia, en nuestra opinión y dada la amplitud del período de análisis, no es obstáculo para detectar con nitidez los principales cambios estructurales sucedidos en las dos últimas décadas.

El trabajo se estructura en cinco apartados. El primero se ocupa de evaluar el crecimiento económico regional a través de las magnitudes de producción, renta y empleo poniendo de relieve un notable paralelismo con la economía española y, por tanto, la ausencia de convergencia con la media nacional. El segundo apartado pasa a ocuparse de los rasgos diferenciales más sobresalientes de la economía

* Area de Economía Aplicada. Departamento de Economía aplicada de la Universidad de Jaén. (1992).

1. A este respecto puede consultarse, entre otros, los trabajos de Higgins y Savoie (1995) y de Levine y Renelt 
andaluza, a través de la compartimentalización en dos etapas del desarrollo económico de la región. En el tercer apartado se detallan los principales cambios de los factores productivos. En cuarto lugar, se aborda el desarrollo experimentado por la estructura productiva andaluza a escala sectorial. Finalmente se aportan las conclusiones más destacadas de nuestro trabajo, poniéndolas en relación con las prescripciones de la teoría económica sobre el crecimiento económico a largo plazo.

\section{EL CRECIMIENTO ECONÓMICO ANDALUZ A TRAVÉS DE LAS MAGNITUDES DE PRODUCCIÓN, RENTA Y EMPLEO}

Nuestro objetivo en este apartado se circunscribe a realizar algunos apuntes de las principales macromagnitudes regionales para conocer su evolución durante las dos últimas décadas. Los parámetros más utilizados, son la producción -que mide la capacidad de un espacio geográfico para generar bienes y servicios- y la renta -que hace referencia a la capacidad adquisitiva de los agentes económicos de la región. Este análisis resulta sugerente en la medida en que los niveles de crecimiento de estas variables tienen incidencia directa en los niveles de ocupación y de bienestar económico de la sociedad en su conjunto.

La primera referencia se realiza a través del cuadro I en el que se recogen los datos del Producto Interior Bruto al coste de los factores (PIB c.f.) y la Renta Familiar Neta Disponible (RFND) de las distintas provincias andaluzas. Los datos proceden de las estimaciones de distribución provincial de la renta efectuadas por el BBV para los años 1975 y 1995 que ofrecen, en nuestra opinión, una imagen de la región suficientemente ilustradora del cambio producido. El PIB de Andalucía en 1995 alcanzó la cifra de 8,9 billones de pesetas, lo que representa el 12,67 por 100 del PIB nacional. Por su parte, la RFND supone el 14,09 por 100 del total nacional. Una primera aproximación a la situación de desventaja de Andalucía se obtiene si comparamos estos valores relativos con el peso de la población andaluza en el total nacional, que alcanza el 18,05 por 100. Estamos, pues, ante una región con bajo nivel productivo y generador de renta en valores agregados con respecto a la media nacional, que mejora sensiblemente su posición si el cálculo se efectúa sobre la RFND, gracias al efecto positivo de los mecanismos de solidaridad interterritorial (Cuadro I).

Como puede observarse, la variación media del PIBcf de las provincias andaluzas, en pesetas de 1995 , es del 78,42 por 100, es decir, 9,39 puntos superior a la experimentada por la media nacional, destacando el fuerte crecimiento alcanzado por Almería (120,75 por 100) y Málaga (114,40 por 100). En el extremo opuesto se sitúa Huelva con un aumento del 20,62 por 100 . Esta favorable evolución de algunas provincias se explica, en parte, por la creciente importancia, tanto en cantidades como en precios, adquirida en este período por la actividad turís- 
tica y algunas producciones agrarias, en detrimento de la presencia relativa de otras provincias con mayor nivel de industrialización como Huelva, Cádiz y Sevilla.

La negativa evolución experimentada en el campo de la actividad industrial cobra mayor relevancia si tenemos en cuenta que estas mismas provincias ya experimentaron una importante pérdida de capacidad productiva ${ }^{2}$ en el período $1955-$ 1975.

\section{CUADRO I}

PRODUCTO INTERIOR BRUTO AL COSTE DE LOS FACTORES (PIBCF)

Y RENTA FAMILIAR DISPONIBLE (RFND)

(EN MILLONES DE PESETAS DE 1995)

\begin{tabular}{|c|c|c|c|c|c|c|}
\hline & \multicolumn{2}{|c|}{1975} & \multicolumn{2}{|c|}{1995} & \multicolumn{2}{|c|}{$\%$ Var. $1975-1995$} \\
\hline & PIBcf & RFND & PIBcf & RFND & PIBcf & RFND \\
\hline Almería & 295.350 & 278.413 & 651.985 & 526.341 & 120,75 & 89,05 \\
\hline Cádiz & 781.118 & 658.185 & 1.318 .614 & 1.092 .712 & 68,81 & 66,02 \\
\hline Córdoba & 515.500 & 462.629 & 897.542 & 785.395 & 74,11 & 69,77 \\
\hline Granada & 461.987 & 445.713 & 873.974 & 784.334 & 89,18 & 75,97 \\
\hline Huelva & 514.208 & 299.421 & 620.231 & 528.020 & 20,62 & 76,35 \\
\hline Jaén & 427.845 & 407.175 & 688.867 & 632.618 & 61,01 & 55,37 \\
\hline Málaga & 751.387 & 655.313 & 1.610 .956 & 1.273 .895 & 114,40 & 94,39 \\
\hline Sevilla & 1.241 .623 & 1.046 .186 & 2.242 .497 & 1.629 .815 & 80,61 & 55,79 \\
\hline Andalucía & 4.990 .943 & 4.253 .035 & 8.904 .666 & 7.253 .130 & 78,42 & 70,54 \\
\hline España & $\begin{array}{c}41.581 .071 \\
1\end{array}$ & $\begin{array}{c}32.148 .782 \\
2\end{array}$ & $\begin{array}{c}70.285 .765 \\
5\end{array}$ & $\begin{array}{c}51.478 .299 \\
9\end{array}$ & 69,03 & 60,13 \\
\hline
\end{tabular}

Fuente: Renta Nacional y su distribución provincial. BBV y elaboración propia.

Si se calcula el peso relativo de la capacidad productiva de cada provincia andaluza en función de la media nacional, es posible apreciar con claridad que Andalucía presenta una distribución bastante heterogénea. Sevilla, Málaga y Cádiz acaparaban, en 1977, el 6,68 por 100 de la producción del país y el 53 por 100 de Andalucía. Esta situación de liderazgo no sólo se mantiene en 1995 sino que se incrementa, llegando a concentrar el 7,36 por 100 del total nacional y el 58 por 100 del total regional. Estos datos, por sí mismos, ponen de relieve la creciente polarización que ha experimentado la actividad productiva hacia determinados espacios geográficos de la región.

Los valores de RFND, que incorporan el efecto de las prestaciones sociales y transferencias del sector público, han experimentado un crecimiento medio del

2. Véase Pérez y otros (1996). 
70,54 рог 100 , sensiblemente superior al nacional $(60,13$ por 100$)$. Podemos afirmar que el papel redistributivo del sector público, a través de los mecanismos de solidaridad interterritorial, ha contribuido a que la banda de oscilaciones interprovinciales sea sensiblemente inferior al considerar esta magnitud. La variación más elevada en renta disponible se presenta en Málaga y Almería y las tasas más reducidas en Jaén y Cádiz (Cuadro II).

\section{CUADRO II}

POBLACIÓN DE DERECHO EN ANDALUCÍA

\begin{tabular}{lrrrrrrr}
\hline & \multicolumn{2}{c}{1975} & \multicolumn{2}{c}{1995} & \multicolumn{2}{c}{$\%$ s/país } & $\begin{array}{c}\% \\
\text { variación } \\
\end{array}$ \\
\cline { 2 - 7 } & \multicolumn{1}{c}{$\begin{array}{c}\text { Poblac. } \\
\text { Derecho }\end{array}$} & $\mathrm{Hab} / \mathrm{km}^{2}$ & $\begin{array}{c}\text { Poblac. } \\
\text { Derecho }\end{array}$ & $\mathrm{Hab} / \mathrm{km}^{2}$ & 1975 & 1995 & $\begin{array}{c}1975- \\
1995\end{array}$ \\
\hline Almería & 385.599 & 44 & 473.957 & 54 & 1,08 & 1,21 & 22,91 \\
Cádiz & 945.638 & 127 & 1.096 .826 & 147 & 2,64 & 2,80 & 15,99 \\
Córdoba & 717.716 & 52 & 755.408 & 55 & 2,00 & 1,93 & 5,25 \\
Granada & 735.778 & 58 & 806.190 & 64 & 2,05 & 2,06 & 9,57 \\
Huelva & 399.862 & 39 & 447.420 & 44 & 1,12 & 1,14 & 11,89 \\
Jaén & 647.086 & 48 & 632.451 & 47 & 1,81 & 1,61 & $-2,26$ \\
Málaga & 914.059 & 125 & 1.204 .671 & 165 & 2,55 & 3,07 & 31,79 \\
Sevilla & 1.370 .705 & 98 & 1.663 .079 & 118 & 3,82 & 4,24 & 21,33 \\
Andalucía & 6.116 .443 & 70 & 7.080 .002 & 81 & 17,06 & 18,05 & 15,75 \\
España & 35.843 .094 & 71 & 39.221 .442 & 78 & 100,00 & 100,00 & 9,43 \\
\hline
\end{tabular}

Fuente: Renta Nacional y su distribución provincial. BBV y elaboración propia.

Los cambios anteriores, referidos al distinto papel jugado por las provincias andaluzas en materia de crecimiento, deben ponerse en relación con la variación experimentada por la población andaluza en este período. La primera nota que conviene destacar es que, al contrario que en el período 1960-75 en que Andalucía presentó fuertes tasas de emigración, entre 1975 y 1995 el incremento de la población andaluza ha podido actuar como freno al proceso de convergencia económica de Andalucía con España y la $U^{3}$.

Así pues, una variable adicional, que ayuda a entender las tendencias de movilidad interprovincial, es el número de habitantes por kilómetro cuadrado, con un

3. Las expectativas de crecimiento demográfico de la región parecen confirmar esta tendencia, sì tenemos en cuenta que la tasa de crecimiento de la población andaluza en 1996 fue cuatro veces superior a la media española. Por otro lado, la proyección de la población de Andalucía realizada por el Instituto de Estadística de Andalucía para el año 2005 estima la población andaluza en 7.466 .442 personas. 
claro liderazgo de Málaga, Cádiz y Sevilla como polos de atracción poblacionales en estas dos décadas, mientras Huelva, Jaén, Almería y Córdoba están muy por debajo de la media andaluza. Si se tienen en cuenta las características orográficas de Almería, se puede afirmar que las áreas geográficas de Andalucía con menor dinamismo demográfico en el período han sido Huelva, Jaén y Córdoba.

Además, los cambios producidos entre 1975 y 1995 indican que la población de derecho andaluza ha pasado de representar el 17,06 por 100 a alcanzar el 18,05 por 100 del total del país. Esta circunstancia presenta a nivel provincializado notables diferencias, desde el fuerte crecimiento de Málaga -superior al 30 por 100hasta la tasa de variación negativa de Jaén $(-2,26$ por 100) y los bajos niveles de crecimiento de la provincia cordobesa, con su efecto inmediato en el envejecimiento de la población.

En síntesis, en estas dos décadas Andalucía presenta tres provincias con buena evolución en la variable poblacional: Málaga, Almería y Sevilla, mientras que las otras cinco ofrecen un reducido dinamismo, siendo Córdoba y Jaén las peor posicionadas. Si a lo anterior añadimos la evolución comentada en términos de PIB y RFND, resulta fácil deducir la existencia de notables desequilibrios económicos intrarregionales que ponemos de manifiesto a continuación. Como comprobaremos más adelante, este crecimiento de la población ha contribuido al crecimiento de la producción agregada, pero no al aumento de los niveles de producto y renta per cápita.

Una vez expuestos los datos demográficos, de producción y de renta más significativos de Andalucía conviene ponerlos en relación, esta tarea se aborda en el cuadro III. Los datos del PIB y la RFND por habitante, reflejan a la vez la influencia del crecimiento económico -numerador de cada índice- y de la evolución demográfica-denominador. Además, la RFND incorpora el efecto redistributivo derivado de la actuación del sector público a través de parámetros como la presión impositiva, las prestaciones sociales, las subvenciones agrarias y la seguridad social, entre otros.

El resultado de este contraste nos permite concluir que el avance económico regional en el campo de la producción ha sido muy similar al nacional, manteniéndose los niveles de PIB por habitante en torno al 70 por 100 de la media del país, después de dos décadas de importantes cambios en todos los ámbitos. Si el cálculo se realiza en porcentaje de la media de los quince miembros de la Unión Europea (UE-15), se aprecia que este supone en 1995 un 54,4 por 100, frente al 56,9 por 100 de 1975.

Este enorme paralelismo de la región con la media nacional, en dos décadas caracterizadas por enormes cambios en aspectos como la tecnología, la productividad o la redistribución del tejido productivo -puesto de manifiesto en la segunda mitad de este trabajo-, permite constatar que esta transformación no ha sido suficiente para sacar a Andalucía de su situación de atraso, en materia de desarro- 
llo económico. No parece, por tanto, que el modelo neoclásico del crecimiento, que sostiene la convergencia de los niveles de desarrollo regional con el paso de los años, pueda ser aplicable al caso andaluz en las dos últimas décadas. Es más, los procesos migratorios parecen haberse comportado de manera inversa a las previsiones de este modelo. Más adelante haremos referencia a la segunda hipótesis del modelo neoclásico referida al potencial de crecimiento de la productividad.

\section{CUADRO III}

PIB Y RFND POR HABITANTE DE LAS PROVINCIAS ANDALUZAS

(EN PORCENTAJES DE LA MEDIA NACIONAL)

\begin{tabular}{lcccc}
\hline & \multicolumn{3}{c}{1975} & \multicolumn{2}{c}{1995} \\
\cline { 2 - 5 } & PIB & RFND & PIB & RFND \\
\hline Almería & 66,03 & 80,50 & 76,76 & 84,61 \\
Cádiz & 71,20 & 77,60 & 67,09 & 75,90 \\
Córdoba & 61,91 & 71,87 & 66,30 & 79,21 \\
Granada & 54,12 & 67,54 & 60,49 & 74,12 \\
Huelva & 110,85 & 83,49 & 77,36 & 89,92 \\
Jaén & 56,99 & 70,16 & 60,78 & 76,21 \\
Málaga & 70,86 & 79,93 & 74,62 & 80,57 \\
Sevilla & 78,08 & 85,10 & 75,24 & 74,67 \\
& 70,34 & 77,52 & 70,18 & 78,05 \\
Andalucía & 1.160 .086 & 896.931 & 1.792 .024 & 1.312 .504 \\
Total Nacional* $=100$ & & & & \\
\hline
\end{tabular}

* En pesetas constantes de 1995.

Fuente: Renta Nacional de España. Panorámica del crecimiento económico 1960-1996. Fundación BBV y elaboración propia.

La desagregación por provincias permite apreciar una notable contracción de los valores relativos de la producción en Huelva y, en menor medida, en Cádiz y en Sevilla, mientras en el extremo opuesto se detecta un fuerte crecimiento de la actividad productiva almeriense, hasta situarse por encima de la media andaluza, gracias al espectacular desarrollo que ha experimentado en estos años su actividad agraria y al creciente peso de la actividad turística. El débil aumento de la capacidad productiva y el crecimento demográfico sensiblemente superior a la media nacional, son dos de los factores que explican el negativo comportamiento de las primeras. Almería, por su parte, a pesar de haberse fortalecido demográficamente, ha experimentado una notable mejora relativa gracias a unos extraordinarios volúmenes de crecimiento, superiores en un 75 por 100 a los de la media nacional. 
Por lo que se refiere a la solidaridad interregional, los valores contenidos en el cuadro III permiten afirmar que la misma ha crecido suavemente en el período. Sobre todo si tenemos en cuenta que, a pesar de que la RFND presenta una variación menor a la del PIB en términos absolutos, los valores por habitantes reflejan un incremento de 53 centésimas -del 77,52 por 100 en 1975 al 78,05 por 100 en 1995. A nivel provincial, el efecto redistribuidor del sector público ha sido intenso en Cádiz, Córdoba, Huelva y Jaén. No obstante, si después de dos décadas en las que se ha producido un fuerte impulso al "Estado del bienestar" sólo podemos hablar de cambios centesimales en esta magnitud, hemos de concluir que la única salida del atraso económico regional habrá de producirse por la vía de mejoras en la producción y la productividad.

La anterior afirmación cobra mayor relieve si tenemos en cuenta que los niveles de solidaridad interespacial han alcanzado unas cotas difíciles de superar en los próximos años, si queremos hacerlas compatibles con las exigencias que, en materia de endeudamiento público, impone el irreversible proceso de integración económica y monetaria.

Los cambios sucedidos en el período colocan a Huelva y Almería en los primeros puestos de Andalucía en PIB por habitante, con una evolución totalmente opuesta, ya que la primera provincia ha sufrido una fuerte caída y la segunda ha crecido significativamente. En RFND sigue destacando Huelva con valores cercanos al 90 por 100 de la media nacional, lo que indica que esta provincia tiene espacios geográficos con niveles inferiores a esta cifra, junto a otras zonas o comarcas no menos relevantes que presentan con toda probabilidad niveles de renta superiores a la media española.

\section{ETAPAS DEL DESARROLLO ECONÓMICO ANDALUZ Y RASGOS DIFERENCIALES DE LA REGIÓN}

La variación experimentada en el período no ha sido homogénea y cabe distinguir, al menos, dos etapas diferenciales que explican el desarrollo económico andaluz desde 1975. Los cuadros IV, V y VI nos permiten apreciar estos cambios. En síntesis, cabe señalar que la primera etapa, que se extiende hasta 1984 , se caracteriza por una fuerte crisis energética con reducidas tasas de crecimiento del PIB y pérdida de convergencia de Andalucía y España respecto a la Unión Europea.

La segunda etapa se inicia a mediados de los ochenta y llega hasta la actualidad, alcanzando su punto álgido en 1989, lográndose en este período una fuerte recuperación y consolidación del crecimiento ${ }^{4}$. Esta segunda etapa permite recu-

4. Aunque el carácter fnndamentalmente descriptivo de nuestro trabajo nos lleva a plantear inicialmente ésta sencilla compartimentalización cronológica, somos conscientes de la existencia de subetapas qne permitirian abundar en algunos de los rasgos diferenciales que se destacan. 
perar parte de las posiciones perdidas en el período anterior. Se trata de una evolución aproximada de una década de la economía andaluza que coincide con los parámetros mostrados por la economía española, hasta tal punto que coincidimos con Aurioles y Velasco $(1995 ; 69)$ cuando sostienen “...que el 95 por 100 del comportamiento de la primera está explicado por los mismos elementos que impulsan a la segunda".

Esta similar evolución en términos globales esconde, no obstante, algunos hechos diferenciales. Como podremos comprobar más adelante, el ciclo económico oscila con más intensidad en Andalucía que en la media española, lo que viene a poner de relieve crecimientos más acusados cuando la evolución es expansiva y recesiones más intensas cuando la fase cíclica es descendente.

Si prestamos atención a la tasa de crecimiento del PIB a precios constantes para los períodos 1975-1985 y 1985-1996, los datos obtenidos permiten confirmar la percepción de que en las etapas de crecimiento (1985-1996), a pesar del bache que supuso la crisis del bienio 1992-1993, Andalucía experimenta un ascenso en sus magnitudes de producción superior a la media española y europea $(3,46$ por 100 frente a 3,10 por 100 de España y 2,20 por 100 de Europa) (Cuadro IV).

CUADRO IV

TASA MEDIA ANUAL DE CRECIMIENTO REAL DEL PIB 1975-1996 DE ALGUNAS REGIONES

\begin{tabular}{lcccc}
\hline & \multicolumn{2}{c}{$1975-1985$} & \multicolumn{2}{c}{$1985-1996$} \\
\cline { 2 - 5 } & Tasa media & $\begin{array}{c}\text { Posición Ranking } \\
\text { Nacional }\end{array}$ & Tasa media & $\begin{array}{c}\text { Posición Ranking } \\
\text { Nacional }\end{array}$ \\
\hline Canarias & 4,78 & 2 & 5,25 & 1 \\
Andalucía & 2,45 & 12 & 3,46 & 7 \\
Madrid & 2,89 & 11 & 3,16 & 9 \\
Media Nacional & 2,48 & - & 3,10 & - \\
Cantabria & 1,89 & 16 & 2,20 & 18 \\
País Vasco & 0,18 & 19 & 2,62 & 16 \\
\hline
\end{tabular}

Fuente: Renta Nacional de España. Panorámica del crecimiento económico 1960-1996. Fundación BBV y elaboración propia.

En nuestra opinión, la observación anterior no se contradice con el hecho de que exista un elevado nivel de integración entre la economía andaluza y la española, sino que lo enriquece, al poner de relieve algunas peculiaridades de nuestra región. Así, la etapa 1975-1985 que se inicia con la crisis energética y el cambio político, supone para Andalucía un crecimiento algo inferior a la media nacional 
(2,45 frente a 2,48 de España), si bien el menor aumento de su población en el período explica que se haya producido una variación más elevada en términos per cápita que en términos de desarrollo económico global.

La comparación con las tasas de crecimiento experimentadas por algunas comunidades autónomas, afianza aún más nuestra impresión de la dificultad de la economía andaluza para alejarse de los senderos de crecimiento trazados por la media nacional, mientras que algunas comunidades, como Canarias, parecen haber encontrado su propio camino hacia la convergencia real.

Junto a esta amplia visión, puede resultar de interés conocer cuáles son las tasas de crecimiento que, en los últimos años, está consiguiendo la economía andaluza, por si fueran ilustrativas de cambios de tendencia hacia un mayor dinamismo productivo.

Si centramos la atención en la década de los noventa - Cuadro V-, se aprecia que, durante un período de seis años, sólo se ha conseguido una reducción del diferencial acumulado de crecimiento del 1,5 por 100 con respecto a la Unión Europea y del 0,9 por 100 sobre la media nacional, lo que refleja un dinamismo productivo que varía muy poco con respecto al observado en las dos décadas precedentes.

\section{CUADRO $\mathrm{V}$}

TASA DE CRECIMIENTO REAL DEL PIB 1991-1996

\begin{tabular}{lrrrrc}
\hline & & & & \multicolumn{2}{c}{ Diferencial } \\
\cline { 5 - 6 } & Andalucía & España & UE-15 & $\begin{array}{c}\text { Andalucía- } \\
\text { España }\end{array}$ & $\begin{array}{c}\text { Andalucía- } \\
\text { UE-15 }\end{array}$ \\
\hline 1991 & & & & 0,2 & 1,0 \\
1992 & 2,5 & 2,3 & 1,5 & 0,7 & 0,5 \\
1993 & 1,4 & 0,7 & 0,9 & $-0,9$ & $-1,5$ \\
1994 & $-2,1$ & $-1,2$ & $-0,5$ & 0,4 & $-0,4$ \\
1995 & 2,5 & 2,1 & 2,9 & 0,2 & 0,2 \\
1996 & 3,2 & 3,0 & 2,5 & 0,3 & 1,2 \\
\multirow{2}{*}{ Acumulado } & 2,6 & 2,3 & 1,4 & 0,9 & 1,5 \\
\hline
\end{tabular}

Fuente: Banco de España, Comisión Europea y elaboración propia.

Estos datos ponen de relieve, en nuestra opinión, la enorme dificultad de la economía andaluza para superar, por sus propios medios y en un razonablemente corto período temporal, esta situación de atraso con respecto a las economías más desarrolladas de la Unión Europea.

La evolución de las tasas de producción en la década de los noventa pone también de manifiesto que sigue vigente el fenómeno de la mayor variabilidad de nues- 
tra economía frente a las oscilaciones del ciclo en España y Europa. Es decir, en los años de crecimiento la economía andaluza alcanza puntas más elevadas y en las etapas de recesión la región muestra una menor capacidad para corregir los desequilibrios existentes en la estructura productiva. De este modo, se hace de nuevo patente la necesidad de incorporar la variable demográfica a nuestro análisis.

El cuadro VI se ha elaborado con el propósito de comparar la evolución de la capacidad productiva por habitante de Andalucía con la de otras regiones españolas que destacan, bien por su mejora de posición en el período, bien por el notable deterioro de su capacidad en términos reales. Si conjugamos el crecimiento ecomómico de estas dos décadas con el nivel de convergencia real, mediante la comparación de la tasa de crecimiento del PIB con el índice de PIB por habitante, podemos resaltar dos hechos. El primero de ellos es que Andalucía partía, en 1977, de un nivel de desarrollo inferior a la media de las regiones españolas y ha logrado incrementos de producción en el período superiores a la media nacional. El segundo hecho destacable es que, a pesar de este favorable cambio, la región ha perdido posición relativa en el índice de PIB por habitante -debido a que el mayor crecimiento de su población ha operado en sentido inverso-, pasando en esta ratio del 72,78 por 100 en 1977 al 71,39 por 100 en 1996 .

CUADRO VI

EVOLUCIÓN PIB PER CÁPITA EN TÉRMINOS REALES DE ALGUNAS REGIONES ESPAÑOLAS (1975-1996)

\begin{tabular}{|c|c|c|c|c|c|c|c|c|c|}
\hline & \multicolumn{3}{|c|}{1975} & \multicolumn{3}{|c|}{1985} & \multicolumn{3}{|c|}{1996} \\
\hline & \multicolumn{2}{|c|}{ Indice } & \multirow{2}{*}{$\begin{array}{c}\text { Posición } \\
\text { en } \\
\text { España }\end{array}$} & \multicolumn{2}{|c|}{ Indice } & \multirow{2}{*}{$\begin{array}{c}\text { Posición } \\
\text { en } \\
\text { España }\end{array}$} & \multicolumn{2}{|c|}{ Indice } & \multirow{2}{*}{$\begin{array}{c}\text { Posición } \\
\text { en } \\
\text { España }\end{array}$} \\
\hline & $\begin{array}{c}\text { España }= \\
100\end{array}$ & $\begin{array}{c}\mathrm{UE}= \\
100\end{array}$ & & $\begin{array}{c}\text { España }= \\
100\end{array}$ & $\begin{array}{c}\mathrm{UE}= \\
100\end{array}$ & & $\begin{array}{c}\text { España }= \\
100\end{array}$ & $\begin{array}{c}\mathrm{UE}= \\
100\end{array}$ & \\
\hline Canarias & 78,69 & 61,2 & 14 & 93,33 & 65,1 & 12 & 100,28 & 76,4 & 8 \\
\hline Baleares & 118,52 & 92,2 & 4 & 141,35 & 98,7 & 1 & 147,69 & 112,5 & 1 \\
\hline Andalucía & 73,11 & 56,9 & 18 & 70,85 & 49,5 & 18 & 71,39 & 54,4 & 19 \\
\hline Murcia & 84,96 & 66,1 & 12 & 82,96 & 57,9 & 15 & 78,70 & 60,0 & 16 \\
\hline Cantabria & 103,84 & 80,8 & 8 & 97,44 & 68,0 & 10 & 90,68 & 69,1 & 12 \\
\hline España & 100,00 & 77,8 & - & 100,00 & 69,8 & - & 100,00 & 76,2 & - \\
\hline
\end{tabular}

Fuente: Renta Nacional de España. Panorámica del crecimiento económico 1960-1996. Fundación BBV y elaboración propia.

Puede apreciarse en el cuadro VI que, tanto Baleares -con elevada capacidad productiva inicial- como Canarias -con reducidos niveles-, han experimentado notables mejoras de crecimiento y han elevado su convergencia real con España 
y Europa de modo significativo. En el extremo opuesto, comunidades como Murcia y Cantabria han perdido posiciones en el ranking europeo. La situación de Andalucía es de acercamiento en los períodos de crecimiento al resto de Europa y de alejamiento en las etapas recesivas. De cualquier modo su nivel de convergencia es inferior al de la mayoría de las comunidades de nuestro país, lo que hace que siga manteniéndose como una de las regiones más atrasadas de la Unión Europea.

Si efectuamos la comparación en función de la Renta Familiar Neta Disponible per cápita, el análisis de estas dos décadas da como resultado que nuestra comunidad ocupa el último lugar entre aquellas que han ganado posición neta en el período, con una mejora en torno a los dos puntos porcentuales.

El detalle provincializado de este proceso de crecimiento se desglosa en unos niveles de PIB por habitante superiores a la media andaluza en Huelva -a pesar de la fuerte contracción productiva del período-, Almería, Sevilla y Málaga, mientras que los niveles más reducidos se dan en Jaén, Granada y Córdoba.

Esta ausencia de despegue económico regional se ratifica al comparar la evoIución de la economía andaluza en función del PIB per cápita comparada con la española. En un período de dos décadas la economía andaluza se mantiene paralela a la española con valores que van del 73,11 por 100 en 1977 , al 71,39 por 100 de 1996, lo que pone de relieve que, en lugar de producirse convergencia, se alcanza una ligera pérdida de posiciones de nuestra región frente a la media española en términos netos. A esta misma conclusión llegamos si comparamos la posición de Andalucía en Europa, que ha pasado del 56,9 por 100 en 1977, al 54,4 por 100 del PIB europeo en 1996.

Podemos concluir, por tanto, que la situación andaluza, medida en función de la capacidad productiva en los ejercicios 1977, 1985 y 1996, pone de manifiesto un importante retroceso en la fase recesiva del ciclo, que se ha recuperado en la última década. La situación a principio y final del período evidencia una clara ausencia de convergencia de Andalucía y España con respecto a la Unión Europea. Si la comparación se ciñe a nuestro país la conclusión es la misma: ausencia de convergencia, en PIB per cápita, de Andalucía con respecto a la media nacional en las dos últimas décadas.

Cabe añadir algunas consideraciones adicionales atendiendo al ranking de las provincias andaluzas en estas dos décadas en VAB y RFD per cápita. La situación en función de la capacidad productiva pone de relieve una pérdida de posiciones generalizada hasta 1985, para recuperarse sólo en algunas provincias en la última década. La pérdida neta de capacidad competitiva de nuestra región sobre la media nacional se aprecia con claridad cuando observamos que, en 1977, tres provincias andaluzas se encontraban entre las diez últimas del país -Jaén, Granada y Córdoba-, en 1985 eran cinco provincias y, en 1995, seis de las ocho provincias andaluzas ocupaban este triste segmento. 
El análisis en función de la renta disponible por habitante permite realizar afirmaciones similares: en 1977 había tres provincias situadas entre las diez últimas del país y todas se encontraban a partir del número treinta; en 1995 la situación provincial de Andalucía está claramente deteriorada, en tanto que son seis las provincias que se encuentran entre las diez últimas y todas las provincias andaluzas se sitúan a partir del puesto treinta y cinco.

\section{EL CAPITAL Y EL TRABAJO EN ANDALUCÍA}

Conviene, tras este panorama poco esperanzador, hacer referencia a las variables clave del estudio del crecimiento económico, como son el capital y el trabajo. Utilizaremos, en primer lugar, las estadísticas de capital acumulado y otras variables básicas elaboradas por la Fundación BBV -Cuadro VII- que nos permiten ofrecer una perspectiva de largo plazo para profundizar aún más en las transformaciones económicas de nuestra región en estas dos décadas. En este caso, la disponibilidad de datos nos obliga a restringir el análisis al período 1975-199I.

\section{CUADRO VII}

ANÁLISIS COMPARATIVO DE ALGUNAS MAGNITUDES

(TASAS DE CRECIMIENTO)

\begin{tabular}{llccc}
\hline & & $1975-85$ & $1985-91$ & $1975-91$ \\
\hline Capital privado & Andalucía & 3,38 & 3,56 & 3,45 \\
& España & 2,78 & 3,13 & 2,91 \\
Capital público & Andalucía & 4,60 & 9,48 & 6,40 \\
& España & 4,36 & 7,37 & 5,48 \\
Capital humano* & Andalucía & 8,14 & 7,50 & 7,90 \\
& España & 6,68 & 5,48 & 6,23 \\
Población activa** & Andalucía & 9,86 & 9,91 & 9,88 \\
& España & 8,45 & 6,71 & 7,80 \\
Población ocupada & Andalucía & 6,46 & 11,33 & 8,26 \\
& España & 5,40 & 9,02 & 6,75 \\
Tasa media de paro & Andalucía & 18,97 & 28,22 & 22,30 \\
& España & 12,23 & 18,85 & 14,28 \\
\hline
\end{tabular}

*Población en edad de trabajar con estudios medios y superiores.

*** Con estudios medios y superiores.

Fuente: Capitalización y crecimiento en España y sus regiones 1955-1995. Fundación BBV y elaboración propia. 
La primera consideración a señalar de la observación del cuadro VII es que la tasa media de crecimiento anual del stock de capital privado en España entre 1975 y 1991 fue del 2,91 por 100, mientras que el volumen de capitalización de la economía andaluza en estos años ha sido muy superior (3,45 por 100), presentando un perfil más dinámico. El desglose del período en las etapas 1975-85 y 1985-91 permite destacar que los diferenciales en el proceso de acumulación han sido siempre favorables a Andalucía, siendo estos de mayor intensidad en la etapa recesiva del ciclo que en los momentos de recuperación y auge.

La responsabilidad de estos fuertes ritmos de crecimiento del capital privado en Andalucía corresponde en un elevado porcentaje al sector servicios, que ha tenido un alto protagonismo en la apuesta por la inversión privada de nuestra región.

El cuadro VII también nos facilita la descripción de algunos rasgos básicos del proceso de acumulación de capital público en Andalucía y España. Una primera panorámica del proceso de acumulación de capital público señala que, mientras el mismo creció a una tasa del 5,48 por 100 en España entre 1975 y 1991, la evolución de Andalucía en el conjunto del período fue netamente superior, alcanzando el 6,40 por 100 .

Dos hechos son destacables de la observación de las cifras de capital público. El primero de ellos es que estas tasas de crecimiento son superiores a las correspondientes al capital privado, siendo estos diferenciales especialmente elevados entre 1985 y 1991. El segundo aspecto a señalar es que el crecimiento de esta magnitud ha estado marcado por el ciclo económico, pero la principal influencia en su evolución la ha tenido el ciclo político, que explica gran parte de este comportamiento agregado.

La anterior descripción quedaría incompleta si no se hiciera referencia al capital humano, dado que el crecimiento económico depende de la dotación de factores productivos - capital y trabajo- y de la calidad de los mismos.

La acumulación de capital humano, como el físico, es fruto del ahorro, renunciando al consumo presente para generar mayores rentas futuras mediante la mejora de la cualificación. La información relativa a la población en edad de trabajar con estudios medios y superiores -definida en el cuadro VII como capital humano- pone de manifiesto la notable mejora que ha experimentado la media de cualificación en Andalucía y, en menor medida, en España ${ }^{5}$. Los datos manejados ponen de relieve que esta acumulación de capital humano ha sido un proceso continuo en todo el período, independiente del ciclo económico, con unas tasas algo más elevadas en la primera de las etapas consideradas.

Puesto que la población en edad de trabajar está constituida por aquellos que han cumplido la edad legal e incluye a los jubilados, resulta de interés analizar

5. Hay que hacer notar que los niveles de partida eran en nuestra región sensiblemente inferiores a la media nacional. 
separadamente la población activa. Si tenemos en cuenta que los jubilados tieneı unos niveles de cualificación inferiores, es lógico que la observación de la pobla ción activa -que en este caso también se circunscribe a los titulados medios : superiores- presente mejoras de cualificación más elevadas.

La intensidad de la mejora experimentada en cualificación por la poblacióı activa ha sido muy alta, tanto en la población española como andaluza. Los dife renciales son favorables en todo momento a Andalucía, con márgenes superiore: a los que se observan al analizar el capital humano. En esta línea cabe destaca tres hechos:

Por un lado, la evolución de esta magnitud no parece estar ligada al cick económico, sino que la mejora en este campo es un efecto directo de la pro gresiva implantación del "Estado del Bienestar" en materia de educación : salud.

En segundo lugar, merece la pena constatar un hecho preocupante, como e: la dificultad estructural de la economía andaluza para integrar en el tejido pro ductivo a una parte importante de los activos más jóvenes y mejor cualifica dos que sus mayores. Este rasgo negativo del proceso de crecimiento aparec cuando se observa que la mejora de la cualificación de la población ocupad: ha sido sensiblemente inferior a la de la población activa, lo que constituye ur reflejo de las tasas de paro más elevadas que sufren los más jóvenes a pesa: de sus mejores niveles educativos. Los datos anteriores confirman la necesidac de ampliar la literatura existente mediante nuevas y profundas investigacione: en, al menos, tres líneas: los posibles desajustes entre la oferta educativa y la: necesidades del tejido productivo; las barreras de entrada al mercado de traba. jo; y la capacidad del tejido productivo andaluz para dar cabida al potencia laboral existente.

Por último, hay que señalar que, pese a los mayores niveles acumulativos de capital humano experimentados por Andalucía en la totalidad del período cor respecto a la media nacional, nuestra región presenta, en 1991 -último año con. siderado-, un porcentaje de población en edad de trabajar con estudios medios y superiores más bajo que la media del país (36,13 frente a 40,59 por 100$)$. Está desfavorable situación se repite al calcularla sobre la población activa $(46,66$ por 100 de Andalucía frente a 52,79 por 100 de España) y ocupada (46,09 frent $\epsilon$ a 51,73$)$.

La evolución de la tasa de paro, como resultado del comportamiento conjunte de la población activa y la ocupada, presenta unos incrementos continuados en e período, con un creciente diferencial desfavorable para Andalucía. Se convierte as en la región española con unas tasas de desempleo más elevadas, tanto en la totalidad del período, como en cualquiera de los dos subperíodos. Esta evolución s $€$ 
explica por las mayores tasas de crecimiento experimentadas en la variable población activa frente a la población ocupada y pone de relieve la dificultad para reducir el volumen de desempleo a medio plazo, si atendemos a la evolución prevista de las capacidades productivas de la región en los próximos años. Se hace necesario, por tanto, complementar nuestro análisis con la observación de la incidencia que hayan podido tener las fuertes transformaciones sufridas por la estructura sectorial de la actividad, objetivo que acometemos en el apartado siguiente de este trabajo.

Finalmente, vale la pena señalar, con respecto al capital humano, que en este período se han producido una serie de procesos tales como el desplazamiento de la población a las grandes ciudades -que parece haber perdido vitalidad en los últimos años-, junto a otros que han mostrado una intensidad creciente. Entre estos últimos se encuentra el proceso de expulsión de activos del sector primario, la creciente participación femenina en el mercado laboral, la incorporación de mayor número de jóvenes al mercado de trabajo y, como consecuencia, la elevación de la tasa de paro.

La influencia de estos cuatro últimos elementos en la evolución y configuración actual del mercado laboral andaluz ha sido determinante, debiendo añadirse, en nuestra opinión las modificaciones normativas efectuadas en estas dos décadas en el marco de relaciones laborales. Estos cambios legales han podido modificar algunas barreras de entrada y salida del mercado laboral, afectando también a la actitud de los desempleados hacia la movilidad geográfica y funcional por el coste de oportunidad que lleva aparejado.

\section{EL DESARROLLO ECONÓMICO ANDALUZ A TRAVÉS DE LA ESTRUCTURA SECTORIAL}

El análisis del crecimiento económico ha de tener en cuenta un amplio abanico de factores como son los niveles de producción y productividad, el nivel tecnológico, la acumulación de capital público y privado, el volumen y capacidad del capital humano y las condiciones del mercado de trabajo. A todos estos factores hemos prestado alguna atención en el análisis precedente, nos corresponde ahora realizar una aproximación a la distribución sectorial de la estructura productiva para hacer, de este modo, referencia al grado de desarrollo económico alcanzado.

En las dos últimas décadas la economía andaluza se ha visto inmersa en un proceso de convergencia productiva y orientación de mercados hacia las demás regiones europeas. En este período, y fruto de este proceso, se ha modificado la estructura de los mercados proveedores y de destino, se ha incrementado el grado de apertura económica al exterior, se han producido importantes cambios institucionales y se han elevado sensiblemente los niveles de competencia. Todas 
estas transformaciones han tenido incidencia, en mayor o menor medida, en actividad productiva de la región.

La apertura al exterior de la economía andaluza en este período ha supuest una mayor presión competitiva, de forma que la capacidad adaptativa de las em presas andaluzas ante los cambios tecnológicos y la estructura productiva ha in fluido de modo determinante en el éxito o fracaso de las mismas e incluso de sec. tores económicos completos. Ante esta situación los elementos tradicionales comc son el precio de venta, el coste del producto y el volumen de factores producti. vos, han ido dejando paso a estrategias basadas en la calidad, la tecnología, lá diferenciación y la comercialización del producto. Por este motivo, merece atención destacada en el análisis del entomo andaluz la evolución de la estructura pro. ductiva -cuadro VII -.

CUADRO VIII

VALOR AÑADIDO BRUTO DE LAS RAMAS PRODUCTIVAS EN ANDALUCÍA Y ESPAÑA (1975-1995) (EN PORCENTAJES DEL TOTAL)

\begin{tabular}{llcccc}
\hline & & 1975 & 1985 & 1995 & $\begin{array}{c}\text { Variación } \\
1975-1995\end{array}$ \\
\hline Agricultura y pesca & & & & & $-6,69$ \\
& Andalucía & 16,25 & 13,56 & 9,56 & $-4,91$ \\
\cline { 2 - 3 } Industria & España & 9,72 & 6,40 & 4,81 & $-10,33$ \\
& Andalucía & 24,55 & 18,05 & 14,22 & $-9,68$ \\
\multirow{2}{*}{ Construcción } & España & 31,82 & 26,40 & 22,14 & \\
& Andalucía & 8,07 & 6,78 & 8,62 & 0,55 \\
\multirow{2}{*}{ Servicios } & España & 7,30 & 5,60 & 7,87 & 0,57 \\
& Andalucía & 51,14 & 61,61 & 67,60 & 16,46 \\
& España & 51,16 & 61,60 & 65,18 & 14,02 \\
\hline
\end{tabular}

Fuente: Renta Nacional y su distribución provincial. BBV y elaboración propia.

Como podemos observar en el cuadro anterior, se ha producido un significativo descenso del sector agrícola-pesquero en la producción regional en los últimos años. En efecto, el sector que más peso relativo ha perdido en el período en Andalucía es la agricultura y pesca, seguido de la industria, mientras que las ganancias son recogidas mayoritariamente por las actividades terciarias y, en segundo lugar, por la construcción. Esta evolución muestra la tendencia, aunque tardía, de la economía andaluza a seguir la evolución de las economías nacional y europea, aunque con una mayor especialización relativa en la actividad turística. En térmi- 
nos generales, el peso de los sectores agrícola, construcción y servicios es superior al de estos mismos sectores en la economía española.

Conviene ahora hacer referencia a la distribución sectorial del mercado de trabajo. La comparación de las principales magnitudes del mercado de trabajo andaluz y español, en las últimas dos décadas, presenta sensibles diferencias con respecto a la distribución del VAB. En primer lugar, permite apreciar que nuestra región partía de unos niveles relativos de ocupación en el sector primario superiores al 30 por 100 del total y un diferencial de casi ocho puntos con respecto a la media nacional en 1977, mientras que el VAB de los elementos primarios se situaba en el 16,25 por 100 . Esta situación se invierte al observar la actividad industrial, mientras los niveles relativos de construcción y servicios son similares.

El cuadro IX nos informa que el fuerte proceso expulsor de mano de obra agrícola y la introducción de mejoras tecnológicas de estas dos décadas, han elevado notablemente la productividad de la población ocupada en la agricultura. En 1995 Andalucía presenta un sector primario que emplea al 11,86 por 100 , frente a una participación en el VAB del 9,56. La población ocupada en la industria ha perdido posiciones en el período, mientras la construcción parece haberse estancado en niveles cercanos al 9 por 100. Tras estas dos décadas la caída de ocupación en la agricultura andaluza ha sido proporcionalmente más elevada que en la media nacional, reduciendo el diferencial hasta 3,4 puntos, mientras que la situación de bajo desarrollo industrial se mantiene en diferenciales similares. No obstante, y a pesar de la fuerte contracción experimentada por la población ocupada en el sector primario, se confirma al final del período la superior presencia relativa de los elementos primarios en la economía andaluza.

\section{CUADRO IX}

EVOLUCIÓN DEL MERCADO DE TRABAJO EN ANDALUCÍA Y ESPAÑA

1975

1995

Andalucía España Diferencial Andalucía España Diferencial

Número de activos (miles)

Tasa de ocupación

Distribución del empleo

- Agricultura

- Industria

- Construcción

- Servicios

Tasa de paro

$\begin{array}{rrrrrr}2016,2 & 13.374,6 & - & 2633,3 & 16203,6 & - \\ 86,46 & 94,43 & -7,97 & 68,49 & 78,53 & -10,04 \\ & & & & & \\ 30,68 & 22,91 & 7,77 & 11,86 & 8,44 & 3,42 \\ 18,67 & 26,91 & -8,24 & 13,06 & 20,00 & -6,94 \\ 9,80 & 9,85 & -0,05 & 9,04 & 8,84 & 0,20 \\ 40,85 & 40,32 & 0,53 & 66,04 & 62,72 & 3,32 \\ 13,54 & 5,57 & 7,97 & 31,51 & 21,47 & 10,04\end{array}$

Fuente: Renta Nacional y su distribución provincial. BBV y elaboración propia. 
El sector que ha recogido el empleo de los demás ha sido el de servicios, qut se coloca 4,5 puntos por encima de la media nacional presentando, como notá destacada, una ganancia neta en el período de veintidós puntos porcentuales.

En síntesis, la visión espacial del VAB y el empleo en Andalucía con respectc a la media nacional evidencia que aún hay pendiente un importante trasvase sec. torial en la región.

El resultado de este cambio de dos décadas es una importante transformaciór en las formas de producción, una reducción del peso de los establecimientos pro. ductivos independientes - por el efecto de la concentración y el aumento dimensional-, un aumento de los procesos de redimensionamiento empresarial y unc creciente especialización sectorial. La conclusión que parece afianzarse es que estas transformaciones no parecen, a corto plazo, capaces de generar empleo a los niveles que demanda la sociedad andaluza. Esta conclusión es fácilmente constatable si tenemos en cuenta que el aumento experimentado por la población activa andaluza en estas dos décadas arroja un resultado neto de más de seiscientas mil personas, mientras que el nivel agregado del factor trabajo -población ocupada- solc ha crecido en sesenta mil personas.

Dos factores que tienen una influencia notable en este proceso son el elevadc peso relativo del sector primario y la desarticulación y escasa presencia de las actividades industriales en la región. Esta apreciación puede ser contrastada con la observación de los niveles de productividad sectorial de las distintas provincias de Andalucía.

El cuadro X ofrece una primera impresión de carácter general, como es el bajo índice de eficiencia global del sistema productivo andaluz, es decir, una capacidad de generación de riqueza por empleado inferior al valor medio nacional -en torno al 88,2 por $100-$.

A escala sectorial se observa una elevada participación de la mano de obra en los procesos productivos agrarios. No obstante, los elementos primarios de la economía andaluza, al contrario de lo que pueda pensarse, no presentan una situación de desventaja con respecto al resto de España. Los datos del cuadro X nos informan de una mayor productividad del sector agrario andaluz (4.216) frente a la media nacional (3.056). Estas cifras deben hacernos reflexionar sobre la cuestión de cuál es el sector que debe realizar el mayor esfuerzo de reconversión para sacar a la región de la tradicional situación de atraso que presenta, dado que la producción agraria parece presentar, en general, buenos niveles de competitividad media en la actualidad. Una conclusión válida para todas las provincias andaluzas a pesar de las fuertes oscilaciones entre los excelentes valores de Cádiz y los bajos niveles de Sevilla.

Si atendemos a los valores de productividad media de la actividad industrial, observamos que la situación comparativa se invierte con respecto a lo que hemos comentado del sector primario. Así, los datos de productividad media de Andalu- 
cía (5.644) son sensiblemente inferiores a los de España (6.556). Esta clara situación de desventaja productiva induce a pensar que una parte importante de la responsabilidad del atraso económico de estas zonas se debe a la incidencia del sector industrial, por sus bajos niveles de competitividad frente al resto del país. La excepción a esta regla la constituyen las provincias de Almería, Cádiz y Huelva, mientras que el problema es especialmente grave en Jaén, Málaga y Granada.

CUADRO $\mathrm{X}$

VABc.f. POR EMPLEO (MILES DE PESETAS) (1995)

\begin{tabular}{lccccc}
\hline & $\begin{array}{c}\text { Agricultura } \\
\text { y Pesca }\end{array}$ & Industria & Construcción & Servicios & $\begin{array}{c}\text { Total } \\
\text { Sectores }\end{array}$ \\
\hline Almería & 3.813 & 8.030 & 3.236 & 5.142 & 4.797 \\
Cádiz & 5.252 & 7.488 & 4.080 & 5.164 & 5.384 \\
Córdoba & 4.662 & 5.287 & 5.313 & 4.830 & 4.915 \\
Granada & 3.973 & 4.498 & 4.416 & 4.778 & 4.616 \\
Huelva & 5.184 & 9.959 & 4.521 & 5.076 & 5.483 \\
Jaén & 4.573 & 4.257 & 3.902 & 4.940 & 4.642 \\
Málaga & 3.468 & 3.895 & 4.164 & 6.147 & 5.433 \\
Sevilla & 3.609 & 5.775 & 5.032 & 5.489 & 5.329 \\
Andalucía & 4.216 & 5.644 & 4.391 & 5.325 & 5.143 \\
España & 3.056 & 6.556 & 4.872 & 6.258 & 5.833 \\
\hline
\end{tabular}

Fuente: Renta Nacional de España y su distribución provincial. BBV y elaboración propia.

De lo anteriormente expuesto se deduce que existe una notoria deficiencia para generar focos de іпradiación de nuevas actividades industriales en toda la región, de ahí su dificultad para acercarse a los niveles medios de desarrollo del resto del país. En nuestra opinión, el despegue industrial de Andalucía, en general, es una clave fundamental de su futuro y exigirá ordenar y organizar los esfuerzos en aquellos ámbitos de la actividad industrial que mejor se adecuen a las capacidades productivas y humanas de cada provincia, de modo que se manifiesten como ventajas competitividas sostenibles a medio plazo. El cuadro XI nos proporciona información adicional de la localización de los sectores industriales más relevantes de Andalucía.

Como se aprecia en el cuadro anterior los sectores industriales andaluces más destacados en volumen de producción total, en 1993, son: los productos alimenticios, bebida y tabaco cuya producción se concentra en Sevilla y Cádiz; Energía y agua, también liderado por Cádiz y Sevilla; y el sector de productos metálicos y maquinaria que concentra la mayor parte de su volumen en Sevilla y Málaga. En términos globales, observamos que el 46 por 100 del VAB industrial andaluz en 1993 se localiza en Sevilla (26 por 100) y Cádiz (20 por 100). 


\section{CUADRO XI}

LOS SECTORES INDUSTRIALES ANDALUCES MÁS RELEVANTES EN 1993 (MILLONES DE PESETAS)

\begin{tabular}{|c|c|c|c|}
\hline VABcf & $\%$ & $\mathrm{~s} /$ total & Localización Industrial \\
\hline Energía y agua & 218.931 & 19,7 & Cádiz $28 \%$ y Sevilla $16 \%$ \\
\hline Minerales y metales & 30.074 & 2,7 & Huelva $36 \%$ y Cádiz $31 \%$ \\
\hline Minerales y prod. no metálicos & 80.202 & 7,2 & Sevilla $29 \%$ y Almería $16 \%$ \\
\hline Productos químicos & 70.371 & 6,3 & Sevilla $33 \%$ y Huelva $21 \%$ \\
\hline Prod. metálicos y maquinaria & 128.935 & 11,6 & Sevilla $32 \%$ y Málaga $15 \%$ \\
\hline Material de transporte & 59.843 & 5,4 & Cádiz $49 \%$ y Sevilla $24 \%$ \\
\hline Prod. aliment., beb. y tabaco & 364.839 & 32,9 & Sevilla $28 \%$ y Cádiz $19 \%$ \\
\hline Textil, cuero y calzado & 48.600 & 4,4 & Sevilla $24 \%$ y Málaga $19 \%$ \\
\hline Papel & 42.821 & 3,9 & Sevilla $29 \%$ y Cádiz $19 \%$ \\
\hline Madera, corcho y muebles & 37.171 & 3,4 & Sevilla $24 \%$ y Córdoba $19 \%$ \\
\hline Caucho, plástico y otros & 28.858 & 2,6 & Córdoba $38 \%$ y Sevilla $18 \%$ \\
\hline Total Industria & 1.110 .645 & 100,0 & Sevilla $26 \%$ y Cádiz $20 \%$ \\
\hline
\end{tabular}

También es posible obtener el nivel de especialización industrial de Andalucía en relación a la media española. Este se obtiene calculando el cociente entre e: VABcf generado por la industria en relación a la población de cada espacio geográfico. En 1995 la producción industrial por persona en España era de 383.468 pesetas, mientras que en Andalucía sólo llegaba a 178.882 pesetas.

La interpretación de estos datos sugiere que el índice de especialización industrial, como indicador del nivel relativo de importancia que tiene la industria en la población andaluza, es sólo del 46,7 por 100 de la media española. Nos encontramos, por tanto, ante una región con escaso desarrollo industrial, que es insuficiente en la mayoría de ramas para cubrir las necesidades de bienes de consumo de estas zonas y precisa importar una parte importante de los bienes de equipo e intermedios, desplazando a otros espacios geográficos parte de su valor añadidc potencial y manteniendo una fuerte dependencia de otras áreas productivas más desarrolladas.

\section{CONSIDERACIONES SOBRE EL CAMBIO ECONÓMICO EN ANDALUCÍA}

El balance del desarrollo económico andaluz de las dos últimas décadas permite concluir que, a pesar del notable crecimiento en términos absolutos, los valores relativos con respecto a España y la UE siguen apuntando una situación eco- 
nómica regional de fuerte retraso, con una estructura sectorial que demanda aún cambios significativos y con un mercado laboral singularmente desequilibrado. El análisis realizado permite apuntar a continuación una serie de características que definen la situación de atraso en el desarrollo económico de Andalucía:

En primer lugar, cabe destacar que, tanto a escala regional como provincial y desde la perspectiva de las dos últimas décadas, no se aprecia convergencia en la economía andaluza, con respecto al resto del país ni de la UE-15 en materia de crecimiento. Sí que se aprecia un fuerte nivel de integración de la economía andaluza en la española y un mayor impacto en la región de las distintas fases del ciclo.

En segundo lugar, conviene señalar que nuestra región presenta un nivel de consumo sensiblemente superior a su capacidad productiva, debido a la solidaridad interterritorial y al volumen de transferencias que recibe y que, en 1995, se puede ilustrar con los siguientes datos: Andalucía tiene un VABc.f. que supone el 70,2 por 100 de la media nacional, frente a valores para la misma variable en Baleares y Cataluña de 148,2 y 123,4 respectivamente; la variable que se identifica con el poder de compra-Renta Familiar Bruta Disponible- le permite a Andalucía ganar 10 puntos de consumo respecto a lo que produce, como consecuencia de los mecanismos de compensación interterritorial puestos en marcha en estos años, pasando al 80,6 por 100 , mientras Baleares y Cataluña descienden al 130,7 y 111,4 respectivamente.

En tercer lugar, la evolución de las dos últimas décadas permite concluir que el impulso al desarrollo económico y la convergencia real sólo puede llevarse a cabo a través del estímulo de la capacidad productiva, cuyo dinamismo depende de la distribución sectorial de la actividad y de otras características adicionales del sistema económico.

Este estancamiento en la convergencia de Andalucía con respecto a la media española ${ }^{6}$ y comunitaria pone de relieve una notable dificultad para proseguir, a un ritmo socialmente aceptable, nuestro acercamiento a Europa.

En el ámbito sectorial la economía andaluza ha seguido un proceso de fuerte terciarización con un sector industrial en declive y un sector primario que, pese al notable proceso de contracción en términos relativos, mantiene una influencia muy elevada en la capacidad productiva total y en la configuración de las relaciones intersectoriales de producción y distribución. De este modo, los únicos polos funcionales existentes, a los que se vinculan las actividades manufactureras más significativas, son las actividades agrarias y de construcción.

6. Andalucía en 1996 presenta el PIB per cápita más bajo del país. 
Por otra parte, el factor humano de la región, a pesar de haber mejorado notablemente su nivel de conocimientos y preparación en estas dos décadas, presenta unos grados de cualificación y un nivel de productividad aparente del trabajo inferiores a la media nacional.

Por otro lado y a pesar de los cambios experimentados por la estructura productiva en las dos décadas recientes, la región sigue desequilibrada desde la doble óptica intraprovincial y extraprovincial, siendo aún importantes los déficit en dotación de factores y los cambios pendientes en términos de desarrollo sectorial. Las vías que, en nuestra opinión, mejorarán esta situación en el futuro pasan por la aplicación de políticas de oferta que fomenten la inversión, realicen importantes avances en el ámbito del desarrollo tecnológico; procuren sustanciales mejoras de la productividad de los factores y atiendan a las posibilidades de desarrollo endógeno de cada espacio geográfico.

En términos generales, cabe hablar de dos Andalucías, la del litoral y zonas urbanas, que está más desarrollada, y la interior y rural, con menor nivel de desarrollo, siendo las provincias más atrasadas las de Jaén, Granada y Córdoba.

El análisis realizado sobre la evolución andaluza con respecto a la media nacional y comunitaria en términos económicos, en el amplio espacio de dos décadas, nos permite concluir que los mercados de estos espacios geográficos no son capaces, por sí mismos, de reducir a corto plazo los desequilibrios existentes. Sobre esta base, las posibilidades y estrategias de fomento del desarrollo económico andaluz pasan, necesariamente, por considerar las posibilidades de actuación de las distintas administraciones públicas y del sistema financiero en su conjunto, orientando su actuación a crear un marco apropiado para la generación de nuevas actividades, estímulo de las ventajas comparativas, mejora de las condiciones de competitividad y aumento de los esfuerzos conducentes a crear unidades económicas de mayor dimensión que cuenten con suficiente poder económico y financiero.

Se trata de una actuación que debe incardinarse en un entorno económico favorable al crecimiento y a la creación e implantación de una fuerte cultura empresarial, en la que se premie el esfuerzo y la ética del trabajo y donde la capacidad emprendedora se convierta en un importante valor social, dado que este factor está muy directamente relacionado con la disposición para ahorrar, invertir y trabajar. Esta es, en nuestra opinión, la principal carencia de Andalucía en la actualidad.

En síntesis, si al principio de este trabajo nos planteábamos cuál podría ser el modelo de crecimiento que mejor se adaptase a la evolución económica de Andalucía entre 1975 y 1995, podemos afirmar que los resultados obtenidos se apartan de las hipótesis de convergencia del enfoque tradiconal o neoclásico. Por el contrario, los hechos considerados para el período objeto de análisis, se acercan más a las hipótesis de los modernos modelos de crecimiento endógeno, dado que no hemos detectado convergencia en estas dos décadas. 
En definitiva, podemos afirmar que la tasa de crecimiento de la economía andaluza va a seguir dependiendo íntimamente de la evolución nacional en los próximos años, dadas las similitudes en instituciones económicas y políticas, y en la distribución sectorial de la actividad productiva. Tan sólo algunas políticas de oferta a nivel regional destinadas a incrementar la productividad, la mayor incidencia sobre la apertura económica regional al exterior o la creación de instrumentos financieros ágiles y competitivos, pueden ofrecer algún margen para obtener diferenciales positivos de crecimiento en los próximos años.

\section{BIBLIOGRAFÍA}

DE LONG, B. y SUMMERS, L. (1992): "Equipment investment and economic growth", Brookings Papers on Economic Activity, $\mathrm{n}^{\mathrm{n}} 2$.

DORNBUSCH, R. y FISCHER, S. (1994): Macroeconomía, McGraw-Hill, Madrid.

ESTEBAN, J. y VIVES, X. (dir.) (1994): Crecimiento y convergencia regional en España y Europa, Instituto de Análisis Económico, CSIC, Fundación de Economía Analítica.

GARCÍA, B., RAYMOND, J.L. y VILLAVERDE, J. (1995): "La convergencia de las provincias españolas", Papeles de Economía Española n" 64, pg. 38-53.

HIGGINS, B: y SAVOIE, D. (1995): Regional development theories and their application, Transaction Publishers, New Jersey.

LEVINE, R. y RENELT, D. (1992): "A sensitivity analysis of cros-country growth regressions", American Economic Review, septiembre.

PÉREZ, F. y OTROS (1996): Capitalización y crecimiento en España y sus regiones 1995-1995, Fundación BBV. Bilbao.

RAYMOND, J.L. (1995): Convergencia real de España con Europa y disparidades regionales en España, Fundación Fies, mimeo.

RAYMOND, J.L. y GARCÍA, B. (1994): "Las disparidades en el PIB per cápita entre las comunidades autónomas y la hipótesis de convergencia", Papeles de Economía Española $\mathrm{n} 1$ 59, pág. 37-58.

VILLAVERDE, J. (1995): Desigualdades provinciales en España, 1955-1991. Doc. De Trabajo $n^{0} 5$, Dpto. de Economía, Universidad de Cantabria. 